Revista

\title{
Multi-Ensayos
}

Vol. 7, núm. 14

ISSN: 2412-3285

https://multiensayos.unan.edu.ni

DOI: https://doi.org/10.5377/multiensayos.v7i14.12006

\section{La Universidad: entre las incertidumbres racionales del presente y la mirada visionaria del futuro}

\section{The University: Between the rational uncertainties of the present and the visionary gaze of the future}

Eduardo López Herrera

Recibido: 28 de junio de 2021. Aceptado: 05 de julio de 2021

\section{RESUMEN}

La universidad, en este cambio de época que estamos viviendo, juega un papel clave dado el vertiginoso desarrollo del conocimiento y su temprana obsolescencia, de ahí que, el aprendizaje debe ser permanente, para de esa manera enfrentar las incertidumbres racionales del presente y la complejidad de este sistema mundo, que permita responder a esa complejidad que la universidad, bien posicionada de su encargo social, puede transformar. El enfrentar las incertidumbres, el tener una mirada visionaria, implica preguntarse ¿Qué universidad tenemos que imaginar y construir para el futuro? Es una reflexión que la comunidad universitaria debe realizar con mucha responsabilidad; para de esa manera, encontrar respuestas pertinentes que posicionen a la universidad en este siglo XXI, como una institución que como bien público, debe dar un invaluable aporte a la sociedad. Un siglo XXI centrado en el ser humano, es un compromiso insoslayable que la universidad debe refrendar; de ahí que la relación entre lo humano y lo científico, debe ser dialéctico. También la forja de profesionales, desde la máxima Casa de Estudios, debe estar impregnada de valores y educación emocional, dos ejes que se complementarían con la docencia, la extensión y la investigación, convirtiéndose esta última en el quehacer pedagógico de la universidad. En el Bicentenario de la Independencia de Centroamérica, lema de nuestra universidad en este 2021, el espíritu de Rubén Darío y Leonel Rugama, son guías del quehacer universitario en los campos de las letras, del compromiso revolucionario y del sentido de patria y de nación.

Palabras claves: Universidad; incertidumbres; humanismo; investigación.

\section{ABSTRACT}

The university, in this change of era that we are experiencing, plays a key role given the vertiginous development of knowledge and its early obsolescence; hence, learning must be permanent, in order to face the rational uncertainties of the present and complexity of this world system, which allows responding to that complexity that the university, well positioned in its social role, can transform. Facing uncertainties, having a visionary gaze, implies asking what university do we have to imagine and build for the future? It is a reflection that the university community must carry out with great responsibility; In order to thus find relevant answers that position the university in this 21st century, as an institution that, as a public good, must make an invaluable contribution to society. A 21st century centered on 1 Docente de Facultad Regional Multidisciplinaria, Estelí. UNAN-Managua/FAREM-Estelí. Correo electrónico: edujlopez9@gmail. com

(c) 2021 Revista Multi-Ensayos. 
the human being is an unavoidable commitment that the university must endorse; hence the relationship between the human and the scientific must be dialectical. Also, the forging of professionals, from the highest House of Studies, must be impregnated with values and emotional education, two axes that would be complemented with teaching, extension and research, the latter becoming the pedagogical task of the university. On the Bicentennial of the Independence of Central America, the motto of our university in this 2021, the spirit of Rubén Dario and Leonel Rugama, are guides of the university endeavor in the fields of letters, the revolutionary commitment and the sense of homeland and nation.

keywords: University; uncertainties; humanism; research.

\section{INTRODUCCIÓN}

El cambio de época que estamos viviendo invita, desde la máxima casa de estudios, a la reflexión, al análisis, a una interpretación objetiva de la realidad, porque se trata efectivamente de enfrentar las incertidumbres del presente, la complejidad de este sistema mundo, donde todo cambia constantemente, nada permanece estático. Así pues, la universidad es una pieza clave de este cambio de época, siempre y cuando su quehacer este repensándose, reinventándose, posicionándose en el ecosistema del conocimiento, estableciendo juicios entre las verdades absolutas y relativas, proyectando el futuro con visión y responsabilidad.

En tanto, la universidad como bien público, tiene un serio compromiso con la sociedad. Se debe a ella, por lo tanto, su esfuerzo, en el contexto en el que nos encontramos es mayúsculo, porque se trata de insertarse en un mundo complejo, de incertidumbres, pero de encontrar las respuestas que respondan a esa complejidad, a esa realidad interdisciplinaria, que la universidad, bien posicionada de su encargo social, puede transformar.

Es importante resaltar, que, en América Latina, son más de trecientos años, desde la fundación de la primera universidad en 1551 en Perú, llamada "Real y Pontificia Universidad de Lima". Por más de cuatrocientos años hasta la fecha, la universidad ha impulsado cambios significativos, que la ubican como una institución clave en el desarrollo de la ciencia y de la cultura en general, dentro de esos cambios; destaca la Reforma Universitaria de Córdoba de 1918, que movió los cimientos de una universidad que estaba desvinculada de la sociedad. Pero lo que acontece en este nuevo siglo, es un cambio profundo en todos los órdenes de la vida, incluido desde luego el conocimiento que se está volviendo obsoleto en muy poco tiempo y se requiere de cambios paradigmáticos, para que la universidad no se vea rebasada y quede como pieza de museo.

Ahora bien, ¿Qué universidad tenemos que imaginar y construir para el futuro? La respuesta no es sencilla, pero tampoco imposible de responder. Esta nueva institución, que se tiene que transformar, no renovar; enfrenta hoy en día, la complejidad no solo de un sistema mundo con serias contradicciones, un planeta amenazado por el cambio climático y las pandemias, una enorme desigualdad social y un desarrollo tecnológico que podría poner de rodillas a la especie humana; si la cuarta revolución industrial, que tiene como punta de lanza a la inteligencia artificial y las que vengan, no se sepan administrar y nos volvamos autómatas, controlados digitalmente. 
El contexto contemporáneo es un mundo de incertidumbres; y lo es. Puesto que, vivimos una época cambiante, donde tenemos que comprender la incertidumbre de lo real, es decir, ser realista en el sentido complejo. Explicar los fenómenos y procesos del mundo contemporáneo que se caracterizan por sus inestabilidades, fluctuaciones, incertidumbres, crisis y emergencias. En tanto, como institución, la universidad, debe preparase para enfrentar lo inesperado, actuar sabiamente, con iniciativa creadora, con visión futurista, para responder a la enorme responsabilidad que tiene en este siglo de inflexión entre conquistar un futuro promisorio o quedarse en el pasado viendo pasar a la historia.

\section{Enfrentando las incertidumbres}

Frente a un mundo de cambios vertiginosos, la universidad se tiene que replantear su quehacer. No es que su aporte al desarrollo científico-social haya sido en vano, todo lo contrario, es invaluable; no obstante, este siglo requiere de un nuevo posicionamiento ante los cambios que se han venido experimentando en los campos de las ciencias físicas, biológicas, en las diferentes tecnologías y en el campo de las ciencias sociales.

Entre el siglo XX y el XXI, la predictividad del futuro se ha hecho imposible, la velocidad del conocimiento en diferentes campos de la ciencia y la misma dialéctica de la realidad, ha implicado que estemos viviendo un cambio de época signado por las incertidumbres.

Al respecto, Edgard Morín plantea:

La ciencia ha creado muchas certezas, pero también muchas incertidumbres. La educación deberá comprender las incertidumbres que han aparecido en las ciencias físicas, biológicas e históricas. Hay que crear principios para enfrentar estratégicamente los riesgos, lo inesperado, lo incierto. Se requiere navegar entre las incertidumbres para anclar en las certezas. La mente humana debe estar preparada para afrontar lo inesperado (como se citó en Acevedo, 2013).

Dicho lo anterior, la universidad, en su filosofía, en sus políticas, debe considerar que la formación de las presentes y futuras generaciones, solo es posible con la puesta en práctica de un currículo flexible, que se adapte a los nuevos tiempos. Porque se hace necesario que una formación holística implique: reaprender a ver, a concebir, a pensar y a actuar.

Por otro lado, los docentes debemos enfrentar, entonces, el desafío de formar a nuestros discentes para un universo complejo de incertidumbres, para un mundo que cambia a diario y que perturba por su inestabilidad. Preparándolos para que asuman un aprendizaje autónomo, que puedan elegir estrategias y aprendan a tomar decisiones. Por eso, las habilidades blandas como la creatividad, perseverancia, adaptabilidad, resiliencia, y manejo del tiempo, son fundamentales en su formación.

Los avances en los campos de la ciencia y en la tecnología misma, como, por ejemplo: nanotecnología, el descubrimiento del genoma humano, física cuántica, antropología, arqueología, inteligencia artificial, etc. por citar algunos de los más importantes; no solo han implicado avances muy significativos para la humanidad, sino que da lugar, por la caducidad y avance del conocimiento, a nuevas hipótesis, a enriquecer la epistemología de la ciencia y enfrentar racionalmente las incertidumbres. 
No hay duda que entramos también en los campos de la filosofía, tan importante en el currículo universitario. No solamente para discutir sobre el origen del universo, sino más bien para adentrarse en la reflexión, en el análisis del porqué cambiar las cosas; por qué establecer nuevos paradigmas en los campos de la ciencia y la cultura y poder enfrentar y encontrar soluciones a las incertidumbres. Pero también potenciar la concepción y la práctica ontológica para patentizar el papel del ser como el centro de este siglo XXI.

Así, enfrentar las incertidumbres, no es obstáculo para una mirada visionaria del futuro por parte de la universidad, todo lo contrario, las incertidumbres son parte de ese repensar de la universidad. No podemos obviar que entre el presente y el futuro se van a generar nuevas incertidumbres; que son parte de un proceso dialéctico de los nuevos tiempos, del inagotable devenir del conocimiento, de las predicciones científicas, de la investigación, que nos debe conducir a respuestas pertinentes que demanda el actual contexto contemporáneo.

\section{La mirada visionaria del futuro}

En esa mirada visionaria, la universidad debe potenciar la relación entre lo global y lo local. Lo global implica una mirada académica-obligatoria del mundo, lo local, es la base para el impulso del desarrollo de nuestros pueblos, también ahí nace nuestro sentido y praxis de identidad, el orgullo patrio, el sentido de nación. La universidad, al expresar una visión globalizadora de toda la realidad y concretamente en el plano internacional, por el contexto globalizante en el que vivimos, debe hacerse sentir ante situaciones tan complejas como: las asimetrías generadas por la globalización, que se expresa en el injusto orden económico internacional y la desigualdad científico-técnica entre los países, el neoliberalismo como expresión de la desigualdad social, el cumplimiento de los ODS, tensiones geopolíticas mundiales, la falta de integración de América Latina, los obstáculos estructurales de la integración centroamericana, etc. Son, entre otros, obstáculos que la universidad debe tener en cuenta en su mirada visionaria, precisamente porque su razón de ser también es universal.

Desde la academia, tenemos que entender que la visión de futuro pasa necesariamente por romper desde ya con saberes profesionalizantes y llevar a la praxis una educación ligada al entorno y que tenga como sustento metodológico y científico, la multi, la interdisciplinariedad y la transdisciplinariedad, para viabilizar un conocimiento pertinente e integral, donde la asimilación de los aprendizajes, con el debido soporte didáctico, formen para la vida.

En tanto, una mirada visionaria implica la flexibilización del currículo, lo que no debe restarle cientificidad a la formación, teniendo como valor agregado las competencias que preparen al nuevo profesional con una sólida e integral formación. Por tanto, el gran reto de la educación del futuro y que la universidad no está al margen de ese reto, no solamente sería enseñar a afrontar las incertidumbres, sino que también saber compartir los nuevos contenidos, los que se verán reforzados por los cambios drásticos y acelerados de las formas de transmisión, sobre todo, en esta coyuntura en que nuestra universidad transita del currículo por objetivos al currículo por competencias. 
Al respecto, la UNAN-Managua (2019) explica

"para lograr una innovación curricular es necesario romper con los modelos de la educación tradicional, la competencia tiene una relación muy grande con la vida, con la profesión, en qué contribuyo a la sociedad, en cómo convierto una tarea en una competencia. Esto es una movilización de conocimientos profundos, nadie aprende oyendo, sino haciendo" (párr.5).

Por lo tanto, una tarea insoslayable con los educandos será fomentarles el talento y la creatividad. Saberlos conducir hacia aprendizajes significativos sin obviar la importancia que hoy tiene el mundo virtual, su uso racional y romper con lo que se llama la sociedad "infoxicada", en la que los estudiantes son incapaces de asimilar el exceso de información que les llega, que, muchas veces es información de desechos y nada sustancial, para su formación científica y en valores.

También no podemos perder la perspectiva que muchos docentes somos inmigrantes digitales, por lo que, tenemos que asumir con mucha responsabilidad pedagógica y didáctica el mundo virtual. La virtuosidad debe ser real nos dice Manuel Castell, de la Universidad Autónoma de Barcelona, yo sostengo que debemos encaminarnos a una mediación pedagógica-virtual-presencial, para conducir metodológicamente los procesos de aprendizaje.

\section{La universidad: entre el humanismo y la cientificidad}

El equilibrio institucional de la universidad, visto desde la perspectiva de la formación de los nuevos profesionales en el presente siglo, lo debe establecer la relación entre el humanismo y la cientificidad. $\mathrm{Ni}$ ciencia pura -que lógicamente hay que cultivarla- alejada de los problemas y soluciones de la sociedad ni humanismo retórico beneficiario y filantrópico que no atienda los problemas estructurales de la sociedad y del ser humano, sobre todo de los más necesitados.

Cientificidad y humanismo como filosofía de la universidad, sobre todo en el actual contexto, impregnado de neoliberalismo; que, aunque se ha demostrado su fracaso, se resiste a morir y ensaya ofensivas para mantener un status quo mundial de enormes asimetrías, que hoy se han profundizado con la pandemia.

La UNAN-Managua (2020), establece:

El sistema universitario debe ser competente y humanizado, a fin de elevar el nivel de vida de las personas, generando cambios en las diferentes áreas: Educación, Salud, Economía, Industria, Gestión de Riesgo, Adaptación al Cambio Climático, Protección a la Madre Tierra, entre otras (párr.3).

Por lo anterior, es de mucha relevancia que el currículo universitario le dé prioridad al tema de los valores y que priorice la concepción y praxis humanista, para que los profesionales, que egresen de las aulas universitarias, se empoderen de los problemas que aquejan a la humanidad como la pobreza, la cuestión étnica, las migraciones, el maltrato a la mujer, violencia sexual, pedofilia, contaminación ambiental, etc. Por eso, hay una referencia importante para la academia y estos son los ODS, los que deben ser un eje transversal en el currículo y poder materializar el humanismo como componente esencial de la formación integral. 
Así, la relación cientificidad y humanismo debe conducir a popularizar la ciencia, a democratizar el conocimiento; para poder impulsar el desarrollo sostenible y que la sociedad en general se apropie de la ciencia y la cultura.

\section{Formación en valores y Educación emocional, dos ejes claves a considerar}

La formación académica, que un estudiante universitario requiere, se ha venido desarrollando a través de los tres ejes claves como son la docencia, la extensión y la investigación y, dentro de esos ejes, como parte de la malla curricular de una asignatura, están las clases generales que se encaminan a la formación de valores en los estudiantes, pero también, en las clases de la especialidad podemos formar valores en los estudiantes; no obstante, muchas veces depende como abordamos la formación de los mismos.

Cabe señalar, que el mundo vive inmerso en una profunda crisis y deterioro de valores que se ha incrementado en la misma medida en que se ha ido afianzando la globalización neoliberal con sus efectos económicos y la agudización de los problemas sociales. Así pues, la educación y consolidación de valores relevantes son una imperiosa necesidad, pues, de la formación y arraigo de los valores en las nuevas generaciones, depende la calidad en la formación integral del egresado universitario y que demanda la sociedad actual para su desarrollo.

En tanto, cuando el profesor socializa con todos, de manera humana y responsable, estamos formando valores, cuando hacemos presente la historia y nos inspiramos en aquellos que fueron protagonistas de la identidad y nacionalidad estamos formamos valores, cuando fortalecemos nuestra identidad local y nacional estamos formando valores, estamos afianzando nuestra identidad y afianzarla implica, ir a Costa Rica o España y no venir hablando como ticos o españoles. La identificación con nuestra casa de estudios, con nuestra universidad, es identidad institucional.

Asimismo, la educación emocional tenemos que irla considerando como un potencial eje, sobre todo, porque la educación emocional es un proceso educativo continuo y permanente, puesto que debe estar presente a lo largo de todo el currículum académico y en la formación permanente a lo largo de toda la vida. Por ello, hay que destacar que los currículos académicos del siglo XX, se centraron, principalmente, en el desarrollo cognitivo, es decir, en la adquisición de conocimientos dejando de lado las emociones; pero hoy sabemos que, para un completo desarrollo de nuestros estudiantes, ambas dimensiones son esenciales y complementarias.

Ahora bien, la inversión en educación emocional podría reflejarse no solo en el rendimiento académico de nuestros estudiantes y en su inteligencia emocional, sino que también a nivel de país en mejor calidad y productividad de sus recursos humanos. ¿Quién prepara esos recursos? Nosotros en la universidad. Así, hoy en día, gran parte del éxito en la escuela en general y en la educación universitaria en particular, lo determina la educación emocional en un alto porcentaje, de ahí, la importancia de su transversalidad. 
Al respecto, Bautista Arrien explica:

Es fácil constatar que una persona motivada aprende antes y mejor que una desmotivada, que un persona emocionalmente equilibrada, tranquila, aprende mejor que una emocionalmente trabada y desbalanceada; que unas relaciones humanas sanas, fluidas armoniosas y comunicativas, generan un clima psicosocial muy propicio para la formación cualitativa y exitosa que se activa en un centro educativo, que la auto estima personal está acompañada de mucha carga emocional, quizá mayor que la propia cognitiva, y que la comunicación se hace vida compartida entre las personas (como se citó en De Castilla, 2017, párr. 34).

Lo referido anteriormente, nos dice que la educación emocional debemos considerarla como eje y como paradigma. Dado el contexto en que nos movemos, de cambios vertiginosos, de complejidades, de incertidumbres racionales, de pandemias, del capitalismo de vigilancia para control de los internautas, en fin, hasta de un colapso civilizatorio que ya está provocando el cambio climático.

Sin temor a equivocarme, la educación emocional es determinante para enfrentar las incertidumbres y para acompañar las nuevas tendencias y enfoques de la educación que nos permita desde la academia vislumbrar un futuro promisorio.

\section{La investigación como quehacer pedagógico de la universidad}

El tránsito al currículo por competencia será exitoso, en la medida que la praxis educativa estéé imbuida del espíritu investigativo, de la curiosidad científica, de la resolución de problemas. Ya no es posible hacer docencia si no está acompañada de la investigación, es la única forma de darle pertinencia a los aprendizajes que se adquieren como parte del proceso de formación de los futuros profesionales.

En un mundo tan cambiante, donde el conocimiento entra en obsolescencia de manera vertiginosa, donde la inteligencia artificial nos acecha, donde la agricultura, la industria, la ganadería, el turismo, hasta la misma medicina, requieren nuevas formas de abordarlas, donde la realidad del medio en que actuamos es interdisciplinaria, se requiere enfocar nuestro quehacer educativo con un carácter holístico, con curiosidad científica, en acciones que problematicen el contenido que estamos desarrollando.

Entonces, hay que empoderarse de la ciencia, profundizar en sus concepciones epistemológicas, sin obviar la popularización de la misma, para aplicarla social y constructivamente. Es decir, garantizar el vínculo social como una tarea impostergable de la universidad pública, de nuestra universidad. Donde practiquemos una ecología de los saberes, como nos dice el académico Boaventura de Souza Santos, de forma tal, que nos permita llegar a los sectores a los que nos debemos, es decir, a los sectores populares.

Debemos de estar claros que, el conocimiento, más aún en nuestra universidad, no es una variable independiente de la sociedad. El saber, los saberes, no deben navegar para hablar en el nuevo lenguaje del mundo virtual, en la nube, por encima de las circunstancias sociales. Hay que investigar, para resolver los problemas de la sociedad, de nuestro pueblo. Asimismo, soy del criterio que las defensas de tesis, sean con el tiempo, más temprano que tarde una gran aula abierta, un aprendizaje para todos, presentarlas en zoom, o en otras plataformas, que los debates de las tesis, sean como diálogos socráticos, que nos enriquezca a todos, que sean lecciones aprendidas. 
Decía Pablo Freire que:

La investigación, la indagación, la búsqueda deben formar parte de la naturaleza de la práctica docente. Lo que se necesita es que el profesor, en su formación permanente, se perciba a sí mismo y asuma que, por ser profesor, es también investigador (como se citó en Parra, 2018, párr.10).

\section{La universidad, entre Darío y Leonel. La universidad con Darío y con Leonel.}

Resulta notorio mencionar, que el compromiso que tenemos como universidad es mayúsculo, si queremos honrar la memoria de estos íconos de nuestra cultura, de nuestra identidad nacional, como son Rubén Darío y Leonel Rugama. Así, la obra de Rubén, es imperecedera, fue una revolución en la lengua que se universalizó, alcanzó un cosmopolitismo de enorme trascendencia que nos llenó de orgullo patrio. Por consiguiente, el patriotismo de Rubén, su concepción nacionalista, su espíritu y compromiso latinoamericanista, todo eso, es parte del valor de su obra, como un invaluable aporte civilizatorio al mundo de las letras y, desde luego, a la humanidad.

Por otro lado, en Leonel Rugama se integran la cultura como símbolo de identidad con la moral revolucionaria, el pensamiento de avanzada con el combatiente de primera línea y la realidad con lo estético, es decir, la revolución.

De tal manera, que, en Darío y Leonel, están los referentes epistemológicos, gnoseológicos y axiológicos que nos alumbran el camino de la ciencia, la cultura y el ser. Tenemos que empoderarnos de estos referentes y trasmitirlos desde su horizontalidad y popularidad a nuestros estudiantes y por ende a la sociedad en general.

Ahora bien, el lema de la universidad en este 2021 es: "Año del Bicentenario de la Independencia de Centroamérica", que el pensamiento y la acción de Rubén y Leonel, su legado, nos inspiren para que el lema cobre vida y sea una escuela de aprendizaje para toda la comunidad universitaria. Por ello, esta Facultad tiene su génesis en la restitución de derechos de la revolución, como una demanda del pueblo segoviano y de Estelí en particular por tener su universidad. Por lo tanto, vamos a enfrentar las incertidumbres y visionar el futuro, con la experiencia de 41 años y el entusiasmo cuando nació nuestro centro universitario el 4 de noviembre de 1979, para iniciar las clases y como dijo Ricardo Morales Avilés, no pararemos de andar jamás.

\section{REFERENCIAS}

Acevedo, A (2013). El pensamiento complejo en Edgar Morin. Las2Orrillas. Recuperado de https://www. las2orillas.co/el-pensamiento-complejo-en-edgar-morin/

De Castilla, M (2017). El pensamiento pedagógico de Juan Bautista Arrien. Recuperado de https:// intecjuba.edu.ni/index.php/blog/80-juan-bautista-arrien

Parra, J (2018). La investigación, un nuevo paradigma de enseñanza: Educar en la incertidumbre. Nueva Tribuna. Recuperado de https://www.nuevatribuna.es/opinion/jesus-parra-montero/ investigacion-nuevo-paradigma-ensenanza-educar-incertidumbre/20180904173143155371.html UNAN-Managua (2019). Universidad de La Habana comparte experiencias con nuestros especialistas 
sobre innovación curricular para el desarrollo de competencias. Recuperado de https://www.unan. edu.ni/index.php/relevantes/universidad-de-la-habana-comparte-experiencias-con-nuestrosespecialistas-sobre-innovacion-curricular-para-el-desarrollo-de-competencias.odp

UNAN-Managua (2020). Académicos centroamericanos analizan los contextos y retos para la normalidad educativa. Recuperado de https://www.unan.edu.ni/index.php/relevantes/academicoscentroamericanos-analizan-los-contextos-y-retos-para-la-normalidad-educativa.odp 